\title{
Diallel Analysis and Genetic Diversity of Some Yellow Maize Inbred Lines (Zea mays L.) Using RAPD and SSR Markers
}

\author{
M.R. Rehan and M. M. Kamara ${ }^{* \#}$ \\ Genetics Dept. and *Agronomy Dept., Faculty of Agriculture, \\ Kafrelsheikh University, Kafr El-Sheikh, Egypt.
}

\begin{abstract}
TNFORMATION about combining ability and genetic diversity among maize inbred lines is fundamental in designing future breeding strategies for improving grain yield. Twenty one $F_{1}$ hybrids were generated by crossing seven yellow maize inbred lines in a half diallel mating scheme in 2013 season. The $21 \mathrm{~F}_{1}$ hybrids plus the two check hybrids (SC166 and SC173) were evaluated in a randomized complete block design with three replications during 2014 season, to estimate combining ability effects and identify type of gene action governing the inheritance of grain yield and other important traits. Results showed that both general (GCA) and specific (SCA) combining ability mean squares were highly significant for all the studied characters. The GCA/SCA ratio was more than unity for all the studied traits, except days to $50 \%$ silking, plant height and grain yield, indicating the preponderance of the additive gene effects in the inheritance of these traits. The inbred line $\mathrm{P}_{1}$ appeared to be the best general combiner for earliness, grain yield and its components. The crosses $\mathrm{P}_{1} \times \mathrm{P}_{2}, \mathrm{P}_{3} \times \mathrm{P}_{7}, \mathrm{P}_{4} \times \mathrm{P}_{7}$, and $\mathrm{P}_{5} \times \mathrm{P}_{6}$ had the best SCA effects for grain yield and most of its component traits. The cross $\mathrm{P}_{1} \times \mathrm{P}_{2}$ significantly out-yielded the two check hybrids (SC166 and SC173). The genetic diversity among the seven yellow parental inbred lines was assessed using two types of molecular markers; Random Amplified Polymorphic DNA (RAPD) and Simple Sequence Repeats (SSR). Seven random primers were used in the RAPD reactions, resulting in the amplification of 82 bands with $95.12 \%$ polymorphism. While, eight pairs of SSR primers were used and resulted in 49 fragments with $100 \%$ polymorphism. Genetic similarity among all possible pairs of inbred lines varied from 0.12 to 0.69 , with an average of 0.34 , for RAPD markers, and from 0.01 to 0.56 , with an average of 0.24 , for SSR markers. The similarity matrices for RAPD and SSR data were not significantly correlated $(\mathrm{r}=0.34, \mathrm{p}>0.05)$. Genetic distances based on RAPD and SSR markers were not significantly correlated with $\mathrm{F}_{1}$ hybrids grain yield $(\mathrm{r}=0.02$ and $\mathrm{r}=0.429, \mathrm{P}>0.05$, respectively). Therefore, the parental genetic distance could not be used to predict the grain yield of the $\mathrm{F}_{1}$ hybrids in this study.
\end{abstract}

Keywords: Maize, Combining ability, Genetic distance, RAPD, SSR.

${ }^{\#}$ Corresponding author: Mailing address: Department of Agronomy, Kafrelsheikh University, 33516, Kafr El-Sheikh, Egypt. $\quad$ E-mail: dr_wagid1212@yahoo.com 
Maize (Zea mays L.) is the third important cereal crop worldwide. It is used in human food, fodder and fuel (Ranum et al., 2014). In Egypt, substantial amount of maize is imported for high domestic consumption and one of the major objectives is to increase maize production in order to decrease its import. The identification of parental inbred lines that perform superior hybrids is the most costly and time consuming phase in maize hybrid development. Combining ability analysis is useful in identifying the mode of gene action and valuable to assess the potentiality of inbred lines.

Assessment of genetic diversity and relatedness among breeding materials has a preponderant role in a breeding program. Molecular markers in many crop species including maize have proven valuable in genetic diversity analysis. Their expression, unlike morphological markers, is not affected by environmental factors; hence they reflect the actual level of the genetic difference existed among the genotypes (Legesse et al., 2007). Several DNA marker technologies are available to study genetic diversity in maize. Among them, Random Amplified Polymorphic DNA (RAPD) markers have been widely used in the maize diversity studies probably because they detected a high level of polymorphism in a rapid and simple manner in addition to its low cost (Bruel et al., 2007 and Devi \& Singh, 2011). Simple Sequence Repeats (SSR) or Microsatellites are useful markers as they have a multi-allelic nature, co-dominant inheritance and reproducibility which make it ideal for genetic diversity studies in maize (Akaogu et al., 2012; Sserumaga et al. 2014 and Nyaligwa et al., 2015).

The association between DNA marker-based genetic distance (GD) and $F_{1}$ hybrids performance for grain yield has been studied in maize. Phumichai et al. (2008), Makumbi et al. (2011) and Kamara (2016) showed that the GD was significantly correlated with $\mathrm{F}_{1}$ hybrids grain yield. However, Menkir et al. (2010) and Akaogu et al. (2012) and Kamara \& Rehan (2015) reported insignificant correlation between GD and $F_{1}$ hybrids grain yield. The objectives of this study were to: (1) Estimate combining ability effects and identify type of gene action governing the inheritance of the studied traits, (2) Assess the genetic diversity among the studied maize inbred lines using RAPD and SSR markers and (3) Estimate the correlations of RAPD and SSR genetic distances with $\mathrm{F}_{1}$ hybrids grain yield.

\section{Materials and Methods}

\section{Field experiments}

Seven yellow maize inbred lines, derived from different sources by maize breeding program (Table 1) were crossed during 2013 season in all possible combinations excluding reciprocals. The resultant $21 \mathrm{~F}_{1}$ crosses along with the two check hybrids SC166 and SC173 were evaluated in a randomized complete block design (RCBD) with three replications at the Experimental Farm of the Faculty of Agriculture, Kafrelsheikh University, during 2014 season. Each plot consisted of two ridges of five meters length, $70 \mathrm{~cm}$ width and $25 \mathrm{~cm}$ between the hills. Two kernels were planted per hill and later thinned to one plant per hill.

Egypt. J. Agron. 38, No. 3 (2016) 
The other cultural practices were followed as usual for ordinary maize field in the area. Data were recorded for days to $50 \%$ silking (day), plant height $(\mathrm{cm})$, ear length $(\mathrm{cm})$, ear diameter $(\mathrm{cm})$, ear height $(\mathrm{cm})$, number of rows/ear, number of kernels/row and grain yield (ardab/faddan) adjusted to $15.5 \%$ moisture content (one ardab $=140 \mathrm{~kg}$, one faddan $=4200 \mathrm{~m}^{2}$ ). The ordinary analysis of variance was done according to Steel \& Torrie (1980). Combining ability analysis was done following the procedure of Griffing (1956), method-4, model-1.

TABLE 1. The code, name and pedigree of the parental maize inbred lines.

\begin{tabular}{|l|c|c|}
\hline $\begin{array}{l}\text { Parent } \\
\text { code }\end{array}$ & Name & Pedigree \\
\hline $\mathrm{P}_{1}$ & Inb. 174 & BS-10-1 \\
\hline $\mathrm{P}_{2}$ & Inb. 202 & H-111 \\
\hline $\mathrm{P}_{3}$ & Inb. 203 & Turk 13 \\
\hline $\mathrm{P}_{4}$ & Inb. 236 & Turk-24 \\
\hline $\mathrm{P}_{5}$ & Inb. 239 & Young-6 \\
\hline $\mathrm{P}_{6}$ & Inb. 247 & Sd.7 x GZ.614 BC1 S7Imported MSA 1967 \\
\hline $\mathrm{P}_{7}$ & Inb. 207 \\
\hline
\end{tabular}

\section{Molecular analysis}

DNA isolation

The young leaves were harvested from 15 to 20 seedlings of each inbred line after twenty days from planting and stored at $-80{ }^{\circ} \mathrm{C}$. Genomic DNA was isolated using CTAB method (Doyle \& Doyle, 1990). DNA quantity as well as quality was assessed using NanoDrop spectrophotometer (ND-1000, USA).

\section{RAPD analysis}

Seven decamer RAPD primers were used for RAPD analysis in this study (Table 6). Polymerase chain reaction (PCR) was carried out in $20 \mu$ reaction mixture containing $1 \mathrm{X}$ Taq buffer, $\mathrm{X} \mathrm{mM}$ dNTPs, $0.5 \mu \mathrm{M}$ primer, $1 \mathrm{U}$ of Taq polymerase and $1.0 \mu \mathrm{l}$ of template DNA. The PCR reaction consisted of an initial denaturation for 2 min at $94^{\circ} \mathrm{C}$, followed by 35 cycles consisting of denaturation at $94^{\circ} \mathrm{C}$ for $20 \mathrm{sec}, 20 \mathrm{sec}$ of annealing at $30^{\circ} \mathrm{C}$, extension of $3 \mathrm{~min}$ at $72^{\circ} \mathrm{C}$ and a final extension of $3 \mathrm{~min}$ at $72{ }^{\circ} \mathrm{C}$.

\section{SSR analysis}

Eight specific maize microsatellite markers (SSR) were used to carry out the SSR analysis (Table 6). PCR was performed in a volume of $10 \mu$ reaction mixture containing $1 \mu \mathrm{L}$ of $20 \mathrm{ng} / \mu \mathrm{L}$ genomic DNA template, 1 unit Taq DNA polymerase (Promega, USA), $2 \mathrm{mM} \mathrm{MgCl} 2,0.2 \mathrm{mM}$ dNTPs and $0.5 \mu \mathrm{M}$ of reverse and forward primer. The PCR reaction was initially started by denaturation at $94^{\circ} \mathrm{C}$ for $2 \mathrm{~min}$, followed by 35 cycles consisting of denaturation at $94^{\circ} \mathrm{C}$ for $30 \mathrm{sec}, 30 \mathrm{sec}$ of annealing at $55^{\circ} \mathrm{C}, 30 \mathrm{sec}$ of extension at $72^{\circ} \mathrm{C}$ and a final extension of $3 \mathrm{~min}$ at $72^{\circ} \mathrm{C}$. Amplified products of both RAPD and SSR analysis were electrophoresed on $1.5 \%$ agarose gel. The gels were stained with ethidium bromide and then distained with tap water and photographed using gel documentation system (UVITEC, UK). 
Data analysis

RAPD and SSR gels were scored as presence (1) and absence (0) of each band for each inbred line for all tested primers. Similarity coefficient matrices were calculated using the Jaccard similarity algorithm (Jaccard, 1908) using PAST program. Cluster analysis was performed to produce a denderogram using method of unweighted pair-group with arithmetic averages (UPGMA).

\section{Results and Discussion}

\section{Analysis of variance}

The mean squares due to genotypes $(\mathrm{G})$ were partitioned into crosses $(\mathrm{C})$, checks (Ch) and $\mathrm{C} v s$. Ch. Genotypes (G) and crosses (C) mean squares were found to be highly significant for all the studied traits, indicating a wide diversity among the genetic materials used in the present study. The differences between the two check hybrids $(\mathrm{Ch})$ were not significant for all the studied traits. Mean squares for crosses vs. checks (C vs. Ch.) were significant for all the measured traits, except ear height and ear diameter. Results in Table 2 showed that both general (GCA) and specific (SCA) combining ability mean squares were highly significant for all the studied characters. These results would suggest the importance of both additive and nonadditive gene effects in determining the performance of these characteristics. However, the magnitude of GCA/SCA ratio exceeded the unity for ear height, ear length, ear diameter, No. of rows/ear and No. of kernels/row, indicating the greatest role of the additive type of gene action in the inheritance of these traits. These results are in accordance with those obtained by Badu-Apraku \& Oyekunle (2012), Abd ElMottalb et al. (2013) and Mousa (2014) for ear height; Hefny (2010) for ear length; El-Badawy (2013) and Ibrahim (2014) for No. of rows/ear and Abo El-Haress (2015) and Kamara (2015) for ear diameter and No. of kernels/row. On the other hand, GCA/SCA ratio was less than unity for days to $50 \%$ silking, plant height and grain yield, indicating that these traits were predominantly controlled by the non-additive type of gene action. These findings are in agreement with those of Ahmed (2013), El-Ghonemy (2015) and Hassan et al. (2016) for plant height and Dodiya \& Joshi (2002), Attia et al. (2015) and Hassan (2015) for days to 50\% silking and grain yield.

TABLE 2. Mean squares from ordinary and combining ability analysis for all the studied traits.

\begin{tabular}{|c|c|c|c|c|c|c|c|c|c|}
\hline & df & $\begin{array}{c}\text { Days } \\
\text { to } 50 \% \\
\text { silking }\end{array}$ & $\begin{array}{c}\text { Plant } \\
\text { height } \\
(\mathrm{cm})\end{array}$ & $\begin{array}{c}\text { Ear } \\
\text { height } \\
(\mathbf{c m})\end{array}$ & $\begin{array}{c}\text { Ear } \\
\text { length } \\
(\mathrm{cm})\end{array}$ & $\begin{array}{c}\text { Ear } \\
\text { diameter } \\
(\mathrm{cm})\end{array}$ & $\begin{array}{c}\text { No. of } \\
\text { rows/ear }\end{array}$ & $\begin{array}{c}\text { No. of } \\
\text { kernels/ } \\
\text { row }\end{array}$ & $\begin{array}{l}\text { Grain } \\
\text { yield } \\
\text { (ard/ } \\
\text { fad) }\end{array}$ \\
\hline Genotypes (G) & 22 & $22.71 * *$ & $521.01 * *$ & $184.14 * *$ & $14.51 * *$ & $0.13 * *$ & $2.67 * *$ & $62.89^{* * *}$ & $29.99 * *$ \\
\hline $\mathrm{F}_{1}$ Crosses $(\mathrm{C})$ & 20 & $24.53^{* * *}$ & $507.77 * *$ & $191.03^{* * *}$ & $12.42^{* * *}$ & $0.13^{* *}$ & $2.70 * *$ & $64.39^{* * *}$ & $31.14 *$ \\
\hline GCA & 6 & $17.47 * *$ & $481.21 * *$ & $287.32 * *$ & $19.63^{* *}$ & $0.25^{* *}$ & $3.81^{* *}$ & $74.71^{* *}$ & $14.69 * *$ \\
\hline $\mathrm{SCA}$ & 14 & $27.56^{* * *}$ & $519.16 * *$ & $149.76^{* * *}$ & $9.33 * *$ & $0.09 * *$ & $2.22 * *$ & $59.96^{* *} *$ & $38.19 *$ \\
\hline Checks (Ch) & 1 & 2.53 & 253.50 & 13.50 & 0.38 & 0.03 & 0.26 & 1.50 & 1.50 \\
\hline Cvs. Ch & 1 & $6.54^{*}$ & $1053.14^{* *}$ & 217.05 & $70.34^{* *}$ & 0.09 & $4.59 * *$ & 94.31 ** & $35.47 *$ \\
\hline Error & 44 & 1.02 & 63.56 & 57.91 & 1.09 & 0.03 & 0.54 & 4.96 & 4.12 \\
\hline \begin{tabular}{|l|} 
GCA/SCA \\
\end{tabular} & & 0.63 & 0.93 & 1.92 & 2.10 & 2.80 & 1.72 & 1.25 & 0.38 \\
\hline
\end{tabular}

Egypt. J. Agron. 38, No. 3 (2016) 


\section{Mean performance}

Mean performance of the $21 \mathrm{~F}_{1}$ crosses and the two check hybrids SC166 and SC173 for all the studied traits are shown in Table 3. The results showed that the mean values of number of days to $50 \%$ silking ranged from 60.5 days $\left(\mathrm{P}_{5} \times \mathrm{P}_{7}\right)$ to 70.67 days $\left(\mathrm{P}_{1} \times \mathrm{P}_{2}\right)$ with an average of 65.25 days. The seven crosses $\left(\mathrm{P}_{1} \times \mathrm{P}_{4}\right)$, $\left(\mathrm{P}_{1} \times \mathrm{P}_{5}\right),\left(\mathrm{P}_{1} \times \mathrm{P}_{6}\right),\left(\mathrm{P}_{2} \times \mathrm{P}_{3}\right),\left(\mathrm{P}_{2} \times \mathrm{P}_{4}\right),\left(\mathrm{P}_{4} \times \mathrm{P}_{5}\right)$ and $\left(\mathrm{P}_{5} \times \mathrm{P}_{7}\right)$ were significantly earlier than the earliest check hybrid SC166. Earliness in maize is favorable for saving water irrigation and escaping destructive injuries caused by the stem corn borers. Plant height means ranged from $226.25 \mathrm{~cm}\left(\mathrm{P}_{4} \times \mathrm{P}_{5}\right)$ to $270 \mathrm{~cm}\left(\mathrm{P}_{1} \times \mathrm{P}_{6}\right)$ with an average of $244.63 \mathrm{~cm}$. Seven crosses $\left(\mathrm{P}_{1} \times \mathrm{P}_{3}\right),\left(\mathrm{P}_{2} \times \mathrm{P}_{6}\right),\left(\mathrm{P}_{3} \times \mathrm{P}_{4}\right),\left(\mathrm{P}_{4} \times \mathrm{P}_{5}\right),\left(\mathrm{P}_{4} \times \mathrm{P}_{6}\right)$, $\left(\mathrm{P}_{5} \times \mathrm{P}_{6}\right)$ and $\left(\mathrm{P}_{5} \times \mathrm{P}_{7}\right)$ were significantly shorter than the shortest check hybrid SC166. Meanwhile, sixteen crosses were significantly shorter than the check hybrid SC173. As for ear height, two crosses $\left(\mathrm{P}_{1} \times \mathrm{P}_{5}\right)$ and $\left(\mathrm{P}_{4} \times \mathrm{P}_{5}\right)$ had significantly lower ear placement compared with the lower check hybrid SC166, and the crosses means ranged from $121 \mathrm{~cm}\left(\mathrm{P}_{4} \times \mathrm{P}_{5}\right)$ to $153 \mathrm{~cm}\left(\mathrm{P}_{1} \times \mathrm{P}_{7}\right)$ with an average of $129.21 \mathrm{~cm}$. Concerning ear length, the crosses mean values ranged from $15.2\left(\mathrm{P}_{2} \times \mathrm{P}_{4}\right)$ to $22 \mathrm{~cm}$ $\left(\mathrm{P}_{1} \times \mathrm{P}_{2}\right.$ and $\left.\mathrm{P}_{1} \times \mathrm{P}_{6}\right)$ with an average of $18.17 \mathrm{~cm}$. None of the crosses significantly surpassed the two check hybrids. Meanwhile, two crosses $\left(\mathrm{P}_{1} \times \mathrm{P}_{2}\right)$ and $\left(\mathrm{P}_{1} \times \mathrm{P}_{6}\right)$ were similar in ear length to the best check hybrid SC173. Regarding ear diameter, the crosses ranged from $4.6 \mathrm{~cm}\left(\mathrm{P}_{3} \times \mathrm{P}_{4}\right)$ to $5.4 \mathrm{~cm}\left(\mathrm{P}_{1} \times \mathrm{P}_{5}\right)$ with an average of $4.9 \mathrm{~cm}$. Only one cross $\left(\mathrm{P}_{1} \times \mathrm{P}_{5}\right)$ gave significantly increased value compared to the best check hybrid SC166. Concerning, number of rows per ear, the crosses ranged from $12.17\left(\mathrm{P}_{4} \times \mathrm{P}_{6}\right)$ to $15.5 \mathrm{~cm}\left(\mathrm{P}_{4} \times \mathrm{P}_{7}\right)$ with an average of 13.79. Four crosses $\left(\mathrm{P}_{1} \times \mathrm{P}_{6}\right)$, $\left(\mathrm{P}_{2} \times \mathrm{P}_{3}\right),\left(\mathrm{P}_{3} \times \mathrm{P}_{7}\right)$ and $\left(\mathrm{P}_{4} \times \mathrm{P}_{7}\right)$ were not significantly different from the best check hybrid SC173. Regarding number of kernels per row, the crosses ranged from 29.0 $\left(\mathrm{P}_{5} \times \mathrm{P}_{7}\right)$ to $45.9\left(\mathrm{P}_{4} \times \mathrm{P}_{7}\right)$ with an average of 38.35 . None of the studied crosses significantly surpassed the highest check hybrid SC173. While, three crosses $\left(\mathrm{P}_{1} \times \mathrm{P}_{2}\right),\left(\mathrm{P}_{1} \times \mathrm{P}_{5}\right)$ and $\left(\mathrm{P}_{4} \times \mathrm{P}_{7}\right)$ did not differ significantly from the check hybrid $\mathrm{SC173}$. Concerning grain yield ( $\mathrm{ard} / \mathrm{fad})$, the crosses mean values ranged from $20.21 \mathrm{ard} / \mathrm{fad}\left(\mathrm{P}_{5} \times \mathrm{P}_{7}\right)$ to $32.46 \mathrm{ard} / \mathrm{fad}\left(\mathrm{P}_{1} \times \mathrm{P}_{2}\right)$ with an average of $25.96 \mathrm{ard} / \mathrm{fad}$. Only the cross $\left(\mathrm{P}_{1} \times \mathrm{P}_{2}\right)$ significantly outyielded the highest yielding check hybrid SC173. Moreover, five and two crosses insignificantly out-yielded the check hybrids SC166 and SC173, respectively.

\section{General combining ability (GCA) effects}

Estimates of GCA effects assigned to each inbred line for all the traits are shown in Table 4 . High positive values of $\left(\hat{g}_{i}\right.$ ) effects would be of importance for all studied traits in question, except days to $50 \%$ silking, plant and ear heights where high negative values would be of interest from the breeder point of view. The results showed that the inbred lines $\mathrm{P}_{1}$ and $\mathrm{P}_{5}$ were the best combiners for earliness, as they had highly significant negative $\left(\hat{g}_{i}\right)$ effects for days to $50 \%$ silking. Meanwhile, the inbred lines $\mathrm{P}_{4}$ and $\mathrm{P}_{5}$ showed significant or highly significant negative $\left(\hat{g}_{i}\right)$ effects for plant height and ear height, indicating that these inbred lines could be considered as a good combiners for developing short 
and lower ear placement hybrids. On the other hand, significant or highly significant positive $\left({ }^{\hat{g}_{i}}\right)$ effects were obtained by the inbred lines $\mathrm{P}_{1}, \mathrm{P}_{2}$ and $\mathrm{P}_{6}$ for ear length; $P_{1}$ and $P_{2}$ for ear diameter; $P_{1}$ and $P_{3}$ for No. of rows/ear; $P_{1}$ and $\mathrm{P}_{6}$ for No. of kernels/row and $\mathrm{P}_{1}$ for grain yield. From the previous results, it could be concluded that the inbred line $\mathrm{P}_{1}$ appeared to be the best general combiner for earliness, grain yield and its components. Attia et al. (2015) and El-

Shamarka et al. (2015), reported desirable and significant $\left(\hat{g}_{i}\right)$ effects for earliness, grain yield and its components in their respective studies.

TABLE 3. Mean performance of the $21 F_{1}$ crosses and the two check hybrids SC166 and SC173 for all the studied traits.

\begin{tabular}{|c|c|c|c|c|c|c|c|c|}
\hline Cross & $\begin{array}{c}\text { Days } \\
\text { to 50\% } \\
\text { silking }\end{array}$ & $\begin{array}{c}\text { Plant } \\
\text { height } \\
(\mathbf{c m})\end{array}$ & $\begin{array}{c}\text { Ear } \\
\text { height } \\
(\mathbf{c m})\end{array}$ & $\begin{array}{c}\text { Ear } \\
\text { length } \\
(\mathbf{c m})\end{array}$ & $\begin{array}{c}\text { Ear } \\
\text { diameter } \\
(\mathbf{c m})\end{array}$ & $\begin{array}{l}\begin{array}{l}\text { No. of } \\
\text { rows/ear }\end{array} \\
\text { res }\end{array}$ & $\begin{array}{c}\text { No. of } \\
\text { kernels/ } \\
\text { row }\end{array}$ & $\begin{array}{c}\text { Grain } \\
\text { yield } \\
(\text { ard/fad })\end{array}$ \\
\hline $\mathrm{P}_{1} \times \mathrm{P}_{2}$ & 70.67 & 245.00 & 129.57 & 22.00 & 5.33 & 14.31 & 45.00 & 32.46 \\
\hline $\mathrm{P}_{1} \times \mathrm{P}_{3}$ & 64.50 & 231.00 & 125.00 & 19.50 & 4.70 & 14.00 & 41.17 & 28.33 \\
\hline $\mathrm{P}_{1} \times \mathrm{P}_{4}$ & 61.50 & 253.75 & 128.75 & 17.30 & 4.80 & 13.33 & 33.80 & 23.25 \\
\hline $\mathrm{P}_{1} \times \mathrm{P}_{5}$ & 63.50 & 251.25 & 121.25 & 18.20 & 5.40 & 14.33 & 44.17 & 25.00 \\
\hline $\mathrm{P}_{1} \times \mathrm{P}_{6}$ & 61.50 & 270.00 & 140.00 & 22.00 & 5.10 & 15.00 & 40.50 & 27.70 \\
\hline $\mathrm{P}_{1} \times \mathrm{P}_{7}$ & 64.30 & 268.75 & 153.00 & 18.80 & 4.90 & 14.00 & 40.83 & 24.00 \\
\hline $\mathrm{P}_{2} \times \mathrm{P}_{3}$ & 60.83 & 266.25 & 137.50 & 18.50 & 5.10 & 15.00 & 36.83 & 23.67 \\
\hline $\mathrm{P}_{2} \times \mathrm{P}_{4}$ & 63.33 & 248.75 & 121.92 & 15.20 & 4.70 & 13.33 & 38.17 & 24.83 \\
\hline $\mathrm{P}_{2} \times \mathrm{P}_{5}$ & 65.00 & 253.75 & 126.25 & 17.50 & 4.90 & 12.67 & 32.00 & 23.33 \\
\hline $\mathrm{P}_{2} \times \mathrm{P}_{6}$ & 67.00 & 227.50 & 122.50 & 19.30 & 5.10 & 13.83 & 40.83 & 25.73 \\
\hline $\mathrm{P}_{2} \times \mathrm{P}_{7}$ & 66.00 & 240.33 & 132.00 & 20.10 & 5.00 & 13.33 & 37.17 & 28.67 \\
\hline $\mathrm{P}_{3} \times \mathrm{P}_{4}$ & 65.50 & 236.25 & 126.50 & 15.60 & 4.60 & 14.50 & 34.67 & 24.17 \\
\hline $\mathrm{P}_{3} \times \mathrm{P}_{5}$ & 66.00 & 240.00 & 125.00 & 15.50 & 4.70 & 13.00 & 36.50 & 25.00 \\
\hline $\mathrm{P}_{3} \times \mathrm{P}_{6}$ & 67.00 & 242.83 & 126.25 & 15.40 & 4.85 & 14.00 & 37.83 & 27.66 \\
\hline $\mathrm{P}_{3} \times \mathrm{P}_{7}$ & 66.00 & 247.50 & 141.25 & 16.20 & 4.90 & 15.33 & 30.33 & 28.93 \\
\hline $\mathrm{P}_{4} \times \mathrm{P}_{5}$ & 63.50 & 226.25 & 121.00 & 18.30 & 4.79 & 13.00 & 36.67 & 25.00 \\
\hline $\mathrm{P}_{4} \times \mathrm{P}_{6}$ & 68.50 & 231.25 & 125.00 & 19.70 & 4.93 & 12.17 & 42.33 & 20.40 \\
\hline $\mathrm{P}_{4} \times \mathrm{P}_{7}$ & 66.80 & 243.75 & 122.00 & 20.00 & 4.70 & 15.50 & 45.90 & 31.00 \\
\hline $\mathrm{P}_{5} \times \mathrm{P}_{6}$ & 69.47 & 238.00 & 130.33 & 19.00 & 4.80 & 13.00 & 39.33 & 30.15 \\
\hline $\mathrm{P}_{5} \times \mathrm{P}_{7}$ & 60.50 & 227.50 & 131.25 & 16.40 & 4.83 & 12.33 & 29.00 & 20.21 \\
\hline $\mathrm{P}_{6} \times \mathrm{P}_{7}$ & 69.00 & 247.67 & 127.00 & 17.00 & 4.70 & 13.67 & 42.33 & 25.58 \\
\hline SC166 & 65.70 & 252.00 & 134.00 & 21.50 & 5.10 & 14.50 & 42.00 & 28.00 \\
\hline SC173 & 67.00 & 265.00 & 137.00 & 22.00 & 4.95 & 14.92 & 43.00 & 29.00 \\
\hline LSD 0.05 & 1.67 & 13.16 & 12.56 & 1.72 & 0.29 & 1.21 & 3.68 & 3.35 \\
\hline LSD 0.01 & 2.23 & 17.60 & 16.80 & 2.30 & 0.39 & 1.62 & 4.92 & 4.48 \\
\hline
\end{tabular}

Egypt. J. Agron. 38, No. 3 (2016) 
TABLE 4. Estimates of general combining ability ( $\hat{g}_{i}$ ) effects of the seven yellow maize inbred lines for all the studied traits.

\begin{tabular}{|l|c|c|c|c|c|c|c|c|}
\hline Inbred line & $\begin{array}{c}\text { Days } \\
\text { to 50\% } \\
\text { silking }\end{array}$ & $\begin{array}{c}\text { Plant } \\
\text { height } \\
(\mathbf{c m})\end{array}$ & $\begin{array}{c}\text { Ear } \\
\text { height } \\
(\mathbf{c m})\end{array}$ & $\begin{array}{c}\text { Ear } \\
\text { length } \\
(\mathbf{c m})\end{array}$ & $\begin{array}{c}\text { Ear } \\
\text { diameter } \\
(\mathbf{c m})\end{array}$ & $\begin{array}{c}\text { No. of } \\
\text { rows/ear }\end{array}$ & $\begin{array}{c}\text { No. of } \\
\text { kernels/ } \\
\text { row }\end{array}$ & $\begin{array}{c}\text { Grain } \\
\text { yield } \\
\text { (ard/fad) }\end{array}$ \\
\hline $\mathrm{P}_{1}$ & $-1.12^{* *}$ & $10.39^{* *}$ & $4.47^{*}$ & $1.76^{* *}$ & $0.17^{* *}$ & $0.44^{*}$ & $3.07^{* *}$ & $1.00^{*}$ \\
\hline $\mathrm{P}_{2}$ & 0.26 & 2.75 & -1.10 & $0.72^{* *}$ & $0.15^{* *}$ & -0.06 & -0.02 & 0.59 \\
\hline $\mathrm{P}_{3}$ & -0.34 & -0.80 & 1.25 & $-1.66^{* *}$ & $-0.11^{*}$ & $0.62^{* *}$ & $-2.55^{* *}$ & 0.41 \\
\hline $\mathrm{P}_{4}$ & -0.48 & $-5.56^{* *}$ & $-6.01^{* *}$ & $-0.58^{*}$ & $-0.17^{* *}$ & -0.18 & 0.29 & $-1.42^{* *}$ \\
\hline $\mathrm{P}_{5}$ & $-0.72^{* *}$ & $-6.21^{* *}$ & $-4.03^{*}$ & $-0.82^{* *}$ & 0.01 & $-0.88^{* *}$ & $-2.49^{* *}$ & $-1.41^{* *}$ \\
\hline $\mathrm{P}_{6}$ & $2.18^{* *}$ & -2.11 & -0.83 & $0.68^{* *}$ & 0.02 & -0.22 & $2.61^{* *}$ & 0.30 \\
\hline $\mathrm{P}_{7}$ & 0.21 & 1.54 & $6.25 * *$ & $-0.10^{* *}$ & -0.07 & 0.28 & -0.91 & 0.53 \\
\hline LSD (0.05) gi & 0.49 & 3.85 & 3.68 & 0.50 & 0.09 & 0.35 & 1.08 & 0.98 \\
\hline LSD (0.01) gi & 0.65 & 5.15 & 4.92 & 0.67 & 0.11 & 0.47 & 1.44 & 1.31 \\
\hline $\begin{array}{l}\text { LSD (0.05) } \\
\text { gi-gj }\end{array}$ & 0.74 & 5.88 & 5.62 & 0.77 & 0.13 & 0.54 & 1.64 & 1.50 \\
\hline $\begin{array}{l}\text { LSD (0.01) } \\
\text { gi-gj }\end{array}$ & 1.00 & 7.87 & 7.51 & 1.03 & 0.18 & 0.72 & 2.20 & 2.00 \\
\hline
\end{tabular}

* and ** significant at 0.05 and 0.01 levels of probability, respectively.

Specific combining ability (SCA) effects

As shown in Table 5, the most desirable significant or highly significant

$\left(\hat{S}_{i j}\right)$ effects were obtained by the crosses $\left(\mathrm{P}_{1} \times \mathrm{P}_{4}\right),\left(\mathrm{P}_{1} \times \mathrm{P}_{6}\right),\left(\mathrm{P}_{2} \times \mathrm{P}_{3}\right),\left(\mathrm{P}_{2} \times \mathrm{P}_{4}\right)$ and $\left(\mathrm{P}_{5} \times \mathrm{P}_{7}\right)$ for days to $50 \%$ silking (towards earliness), $\left(\mathrm{P}_{1} \times \mathrm{P}_{2}\right),\left(\mathrm{P}_{1} \times \mathrm{P}_{3}\right)$, $\left(\mathrm{P}_{2} \times \mathrm{P}_{6}\right),\left(\mathrm{P}_{2} \times \mathrm{P}_{7}\right)$ and $\left(\mathrm{P}_{5} \times \mathrm{P}_{7}\right)$ for plant height (towards shorter plants), $\left(\mathrm{P}_{1} \times \mathrm{P}_{3}\right)$, $\left(\mathrm{P}_{1} \times \mathrm{P}_{5}\right),\left(\mathrm{P}_{4} \times \mathrm{P}_{7}\right)$ and $\left(\mathrm{P}_{6} \times \mathrm{P}_{7}\right)$ for ear height (towards lower ear placement), $\left(\mathrm{P}_{1} \times \mathrm{P}_{2}\right),\left(\mathrm{P}_{1} \times \mathrm{P}_{3}\right),\left(\mathrm{P}_{1} \times \mathrm{P}_{6}\right),\left(\mathrm{P}_{2} \times \mathrm{P}_{3}\right),\left(\mathrm{P}_{2} \times \mathrm{P}_{7}\right),\left(\mathrm{P}_{4} \times \mathrm{P}_{5}\right),\left(\mathrm{P}_{4} \times \mathrm{P}_{6}\right)$ and $\left(\mathrm{P}_{4} \times \mathrm{P}_{7}\right)$ for ear length, $\left(\mathrm{P}_{1} \times \mathrm{P}_{5}\right),\left(\mathrm{P}_{3} \times \mathrm{P}_{7}\right)$ and $\left(\mathrm{P}_{4} \times \mathrm{P}_{6}\right)$ for ear diameter, $\left(\mathrm{P}_{1} \times \mathrm{P}_{5}\right),\left(\mathrm{P}_{1} \times \mathrm{P}_{6}\right)$ and $\left(\mathrm{P}_{4} \times \mathrm{P}_{7}\right)$ for No. of rows/ear, $\left(\mathrm{P}_{1} \times \mathrm{P}_{2}\right),\left(\mathrm{P}_{1} \times \mathrm{P}_{3}\right),\left(\mathrm{P}_{1} \times \mathrm{P}_{5}\right),\left(\mathrm{P}_{3} \times \mathrm{P}_{5}\right),\left(\mathrm{P}_{4} \times \mathrm{P}_{7}\right)$ and $\left(\mathrm{P}_{6} \times \mathrm{P}_{7}\right)$ for No. of kernels/row and $\left(\mathrm{P}_{1} \times \mathrm{P}_{2}\right),\left(\mathrm{P}_{3} \times \mathrm{P}_{7}\right),\left(\mathrm{P}_{4} \times \mathrm{P}_{7}\right)$ and $\left(\mathrm{P}_{5} \times \mathrm{P}_{6}\right)$ for grain yield. These crosses may find prime importance in breeding programs for the traditional breeding procedures. It is notable that the crosses that showed high SCA effects for grain yield also showed high SCA effects for one or more traits of yield components. For example, the cross $\left(\mathrm{P}_{4} \times \mathrm{P}_{7}\right)$ which showed high SCA effects for grain yield also showed high SCA effects for ear length, No. of rows/ear and No. of kernels/row.

\section{Levels of polymorphism}

The genetic diversity within the seven yellow maize parental lines was assessed using two types of molecular markers (RAPD and SSR). The obtained results are presented in Table 6. Seven RAPD primers were used and generated a total of 82 reproducible DNA bands/alleles, of which 78 bands $(95.12 \%)$ were polymorphic (Fig. 1). The number of polymorphic bands detected with each primer ranged from 8 (primer OP-1) to 14 (primer OP-7) with an average of 9.75 bands per primer. The level of RAPD polymorphism observed in the study was higher than those reported in previous studies of maize by Lanza et al. (1997) (80.6\%), Mukharib et al. (2010) (73.02\%) and Molin et al. (2013) (81.9\%). 
TABLE 5. Estimates of specific combining ability $\left(\hat{S}_{i j}\right)$ effects of the $21 \mathbf{F}_{\mathbf{1}}$ crosses for all the studied traits.

\begin{tabular}{|c|c|c|c|c|c|c|c|c|}
\hline Cross & $\begin{array}{c}\text { Days } \\
\text { to } 50 \% \\
\text { silking }\end{array}$ & $\begin{array}{c}\text { Plant } \\
\text { height } \\
(\mathbf{c m})\end{array}$ & $\begin{array}{c}\text { Ear } \\
\text { height } \\
(\mathbf{c m})\end{array}$ & $\begin{array}{c}\text { Ear } \\
\text { length } \\
(\mathrm{cm})\end{array}$ & $\begin{array}{c}\text { Ear } \\
\text { diameter } \\
(\mathrm{cm})\end{array}$ & $\begin{array}{c}\text { No. of } \\
\text { rows/ear }\end{array}$ & $\begin{array}{l}\text { No. of } \\
\text { kernels/ } \\
\text { row }\end{array}$ & $\begin{array}{c}\text { Grain } \\
\text { yield } \\
(\text { ard/fad })\end{array}$ \\
\hline $\mathrm{P}_{1} \times \mathrm{P}_{2}$ & $6.27 * *$ & $-12.78 * *$ & -3.01 & $1.35 * *$ & 0.12 & 0.13 & $3.60 * *$ & $4.91 * *$ \\
\hline $\mathrm{P}_{1} \times \mathrm{P}_{3}$ & 0.70 & $-23.23 * *$ & $-9.93 * *$ & $1.23^{*}$ & $-0.26 * *$ & $-0.85^{*}$ & $2.30^{*}$ & 0.97 \\
\hline $\mathrm{P}_{1} \times \mathrm{P}_{4}$ & $-2.16^{* *}$ & 4.29 & 1.09 & $-2.05 * *$ & -0.10 & $-0.72 *$ & $-7.91 * *$ & $-2.29 *$ \\
\hline $\mathrm{P}_{1} \times \mathrm{P}_{5}$ & 0.07 & 2.44 & $-8.39 *$ & -0.91 & $0.32 * *$ & $0.98 * *$ & $5.23 * *$ & -0.55 \\
\hline $\mathrm{P}_{1} \times \mathrm{P}_{6}$ & $-4.83 * *$ & $17.09 * *$ & 7.16 & $1.39 * *$ & 0.01 & $0.98 * *$ & $-3.54 * *$ & 0.44 \\
\hline $\mathrm{P}_{1} \times \mathrm{P}_{7}$ & -0.05 & $12.19^{* *}$ & $13.07 * *$ & $-1.03^{*}$ & -0.10 & -0.52 & 0.32 & $-3.49 * *$ \\
\hline $\mathrm{P}_{2} \times \mathrm{P}_{3}$ & $-4.34 * *$ & $19.66^{* *}$ & $8.14 *$ & $1.27 *$ & 0.16 & 0.65 & 1.06 & $-3.28 * *$ \\
\hline $\mathrm{P}_{2} \times \mathrm{P}_{4}$ & $-1.70^{* *}$ & 6.92 & -0.18 & $-3.11 * *$ & $-0.18 *$ & -0.22 & -0.45 & -0.30 \\
\hline $\mathrm{P}_{2} \times \mathrm{P}_{5}$ & 0.20 & $12.57 * *$ & 2.17 & -0.57 & -0.16 & -0.19 & $-3.84 * *$ & -1.80 \\
\hline $\mathrm{P}_{2} \times \mathrm{P}_{6}$ & -0.70 & $-17.78 * *$ & -4.78 & -0.27 & 0.03 & 0.31 & -0.11 & -1.12 \\
\hline $\mathrm{P}_{2} \times \mathrm{P}_{7}$ & 0.27 & $-8.59 *$ & -2.36 & $1.31 *$ & 0.02 & -0.69 & -0.25 & 1.59 \\
\hline $\mathrm{P}_{3} \times \mathrm{P}_{4}$ & $1.07 *$ & -2.03 & 2.05 & -0.33 & -0.02 & 0.28 & -1.42 & -0.78 \\
\hline $\mathrm{P}_{3} \times \mathrm{P}_{5}$ & $1.80 * *$ & 2.37 & -1.43 & -0.19 & -0.10 & -0.52 & $3.19 * *$ & 0.05 \\
\hline $\mathrm{P}_{3} \times \mathrm{P}_{6}$ & -0.10 & 1.11 & -3.38 & $-1.79 * *$ & 0.04 & -0.19 & -0.57 & 1.00 \\
\hline $\mathrm{P}_{3} \times \mathrm{P}_{7}$ & 0.87 & 2.12 & 4.54 & -0.21 & $0.18^{*}$ & 0.64 & $-4.56^{* *}$ & $2.04 *$ \\
\hline $\mathrm{P}_{4} \times \mathrm{P}_{5}$ & -0.56 & -6.61 & 1.84 & $1.53 * *$ & 0.06 & 0.28 & 0.52 & 1.87 \\
\hline $\mathrm{P}_{4} \times \mathrm{P}_{6}$ & $1.54 * *$ & -5.71 & 2.64 & $1.43 * *$ & $0.19^{*}$ & $-1.22 * *$ & 1.09 & $-4.43 * *$ \\
\hline $\mathrm{P}_{4} \times \mathrm{P}_{7}$ & $1.81 * *$ & 3.14 & $-7.45^{*}$ & $2.51 * *$ & 0.05 & $1.61 * *$ & $8.17 * *$ & $5.93 * *$ \\
\hline $\mathrm{P}_{5} \times \mathrm{P}_{6}$ & $2.74 * *$ & 1.69 & 5.99 & 0.97 & -0.12 & 0.31 & 0.86 & $5.30 * *$ \\
\hline $\mathrm{P}_{5} \times \mathrm{P}_{7}$ & $-4.25 * *$ & $-12.46^{* *}$ & -0.18 & -0.85 & 0.00 & $-0.86^{*}$ & $-5.96 * *$ & $-4.87 * *$ \\
\hline $\mathrm{P}_{6} \times \mathrm{P}_{7}$ & $1.35 * *$ & 3.61 & $-7.63 *$ & $-1.75^{* *}$ & -0.15 & -0.19 & $2.28 *$ & -1.20 \\
\hline $\operatorname{LSD} 5 \%\left(\mathrm{~s}_{\mathrm{ij}}\right)$ & 0.96 & 7.60 & 7.25 & 0.99 & 0.17 & 0.70 & 2.12 & 1.93 \\
\hline $\operatorname{LSD} 1 \%\left(\mathrm{~s}_{\mathrm{ij}}\right)$ & 1.29 & 10.16 & 9.70 & 1.33 & 0.23 & 0.94 & 2.84 & 2.59 \\
\hline $\begin{array}{l}\text { LSD 5\% } \\
\left(\mathrm{s}_{\mathrm{ij}}-\mathrm{s}_{\mathrm{ik}}\right)\end{array}$ & 1.49 & 11.77 & 11.23 & 1.54 & 0.26 & 1.08 & 3.29 & 2.99 \\
\hline $\begin{array}{l}\text { LSD } 1 \% \\
\left(\mathrm{~s}_{\mathrm{ij}}-\mathrm{s}_{\mathrm{ik}}\right)\end{array}$ & 1.99 & 15.74 & 15.03 & 2.06 & 0.35 & 1.45 & 4.40 & 4.01 \\
\hline $\begin{array}{l}\text { LSD 5\% } \\
\left(\mathrm{s}_{\mathrm{ij}}-\mathrm{S}_{\mathrm{kl}}\right)\end{array}$ & 1.29 & 10.19 & 9.73 & 1.33 & 0.23 & 0.94 & 2.85 & 2.59 \\
\hline $\begin{array}{l}\text { LSD 1\% } \\
\left(\mathrm{s}_{\mathrm{ij}}-\mathrm{S}_{\mathrm{kl}}\right)\end{array}$ & 1.72 & 13.61 & 12.99 & 1.78 & 0.30 & 1.25 & 3.80 & 3.46 \\
\hline
\end{tabular}

Egypt. J. Agron. 38, No. 3 (2016) 
TABLE 6. Details of the selected primers used in RAPD and SSR profiling analysis.

\begin{tabular}{|c|c|c|c|c|c|c|}
\hline $\begin{array}{c}\text { Marker } \\
\text { type }\end{array}$ & $\begin{array}{l}\text { Primer } \\
\text { name }\end{array}$ & Sequence & TNB & NMB & NPB & PPB \\
\hline \multirow{8}{*}{ RAPD } & OP-1 & CCCAAGGTCC & 8 & - & 8 & 100 \\
\hline & OP-2 & CATACCGTGG & 12 & - & 12 & 100 \\
\hline & OP-3 & AGCATGGCTC & 10 & - & 10 & 100 \\
\hline & OP-4 & GACCAATGCC & 15 & - & 15 & 100 \\
\hline & OP-5 & TGAGGGTCCC & 9 & - & 9 & 100 \\
\hline & OP-6 & GGGTCTCGGT & 13 & 3 & 10 & 76.92 \\
\hline & OP-7 & AGAGCCGTCA & 15 & 1 & 14 & 93.33 \\
\hline & \multicolumn{2}{|l|}{ Total } & 82 & 4 & 78 & 95.12 \\
\hline \multirow{9}{*}{ SSR } & phi072 & $\begin{array}{l}\text { F: ACCGTGCATGATTAATTTCTCCAGCCTT } \\
\text { R: GACAGCGCGCAAATGGATTGAACT }\end{array}$ & 2 & - & 2 & 100 \\
\hline & phi024 & $\begin{array}{l}\text { F: ACTGTTCCACCAAACCAAGCCGAGA } \\
\text { R: AGTAGGGGTTGGGGATCTCCTCC }\end{array}$ & 1 & - & 1 & 100 \\
\hline & Umc1014 & $\begin{array}{l}\text { F: GAAAGTCGATCGAGAGACCCTG } \\
\text { R: CCСТCTCTTCACCCCTTCCTT }\end{array}$ & 5 & - & 5 & 100 \\
\hline & phi299852 & $\begin{array}{l}\text { F: GATGTGGGTGCTACGAGCC } \\
\text { R: AGATCTCGGAGCTCGGCTA }\end{array}$ & 1 & - & 1 & 100 \\
\hline & phil12 & $\begin{array}{l}\text { F: TGCCCTGCAGGTTCACATTGAGT } \\
\text { R: AGGAGTACGCTTGGATGCTCTTC }\end{array}$ & 4 & - & 4 & 100 \\
\hline & phi015 & $\begin{array}{l}\text { F: GCAACGTACCGTACCTTTCCGA } \\
\text { R: ACGCTGCATTCAATTACCGGGAAG }\end{array}$ & 3 & - & 3 & 100 \\
\hline & umc 1033 & $\begin{array}{l}\text { F: CTTCTTCGTAAAGGCATTTTGTGC } \\
\text { R: GTGCGGGATTCCTTAGTTTGC }\end{array}$ & 3 & - & 3 & 100 \\
\hline & phi301654 & $\begin{array}{l}\text { F: GAATGCATGCTTTTCAAGGAC } \\
\text { R: CGCACAGAGAGCAGAACG }\end{array}$ & 2 & - & 2 & 100 \\
\hline & \multicolumn{2}{|r|}{ ( } & 21 & & 21 & 100 \\
\hline
\end{tabular}

TNB: Total number of bands; NMB: number of monomorphic bands; NPB: number of polymorphic bands; PPB: percent of polymorphic bands.


Fig.1. DNA-RAPD patterns generated by four arbitrary primers (OP-4, OP-5, OP-6 and OP-7) with the seven inbred lines (P1 - P7). (M) refers to the DNA ladder.

Egypt. J. Agron. 38, No.3 (2016) 
For SSR analysis, eight primer pairs used and all were polymorphic. A total of 21 polymorphic bands/ alleles were amplified with $100 \%$ polymorphism (Table 6 and Fig. 2). This result was in agreement with Sun et al. (2001) and Souza et al. (2008). They also detected $100 \%$ polymorphism within maize inbreds using SSR markers. The number of polymorphic bands detected with each primer ranged from 1 (primers phi024 and phi299852) to 5 (primer Umc1014) with an average of 2.63 bands per primer. The mean number of SSR bands per primer detected in this study was similar to those obtained by Menkir et al. (2004) and Akinwale et al. (2014). However, it was lower than those reported by Nyaligwa et al. (2015) and Pandit et al. (2016).
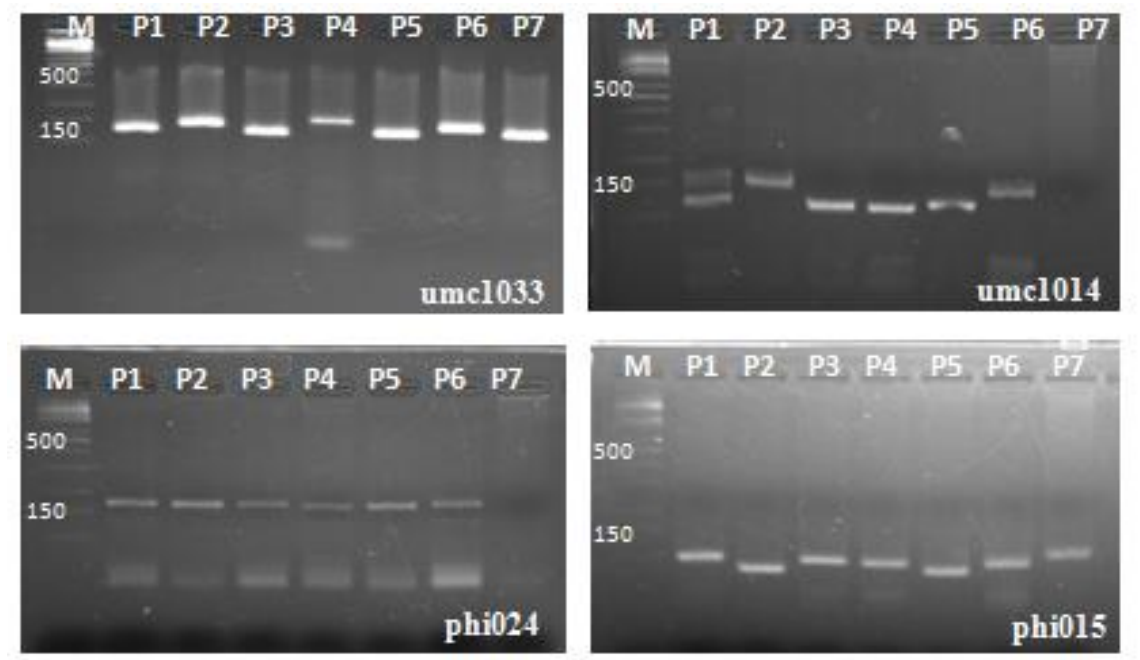

Fig. 2. DNA-SSR patterns generated by four specific primers (umc1033, umc1014, phi112 and phi015) with the seven inbred lines (P1 - P7). (M) refers to the DNA ladder.

The high level of polymorphism observed in this study for both marker types (RAPD and SSR) is consistent with the previous studied of Senior et al. (1998), Souza et al. (2008) and Abdellatif \& Khidr (2010) which reported that the polymorphism levels for different types of molecular markers were high in maize. Souza et al. (2008) found that SSR markers obtained higher levels of polymorphism than RAPD markers in maize. The high level of polymorphism associated with SSR markers is caused by replication slippage and the codominant nature of this marker, which responsible for generating SSR allelic diversity (Pejic et al., 1998 and Souza et al,. 2008).

\section{Genetic distance and cluster analysis}

Two independent genetic similarity matrices were produced for the RAPD and SSR data according to Jaccard's coefficient (Jaccard, 1908). The similarity coefficient for RAPD markers ranged from 0.12 to 0.69 with an average of 0.34

Egypt. J. Agron. 38, No. 3 (2016) 
(Table 7, above diagonal). The lowest genetic similarity (0.12) was detected between $\mathrm{P}_{3}$ and $\mathrm{P}_{7}$ also, obtained between $\mathrm{P} 6$ and P7. Meanwhile, the highest genetic similarity was (0.69) observed between the inbred lines $\mathrm{P}_{2}$ and $\mathrm{P}_{4}$. Based on SSR data, the similarity coefficient ranged from 0.01 to 0.56 with an average of 0.24 (Table 7 , below diagonal). The lowest genetic similarity (0.01) was detected between $\mathrm{P}_{2}$ and $\mathrm{P}_{7}$, whereas the highest genetic similarity was $(0.56)$ observed between the inbred lines P3 and P5. In general, the SSR markers data showed less similarity than those obtained from the RAPD markers. These results corresponded well with the findings of Sun et al. (2001) and Laborda et al. (2005) which showed that the SSR markers had a better ability to investigate diversity, showing higher polymorphism and consequently higher variability.

TABLE 7. Similarity matrices based on RAPD (above diagonal) and SSR (below diagonal) markers among maize inbred lines $\left(\mathbf{P}_{1}-\mathbf{P}_{7}\right)$.

\begin{tabular}{|l|c|c|c|c|c|c|c|}
\hline & $\mathbf{P}_{\mathbf{1}}$ & $\mathbf{P}_{\mathbf{2}}$ & $\mathbf{P}_{\mathbf{3}}$ & $\mathbf{P}_{\mathbf{4}}$ & $\mathbf{P}_{\mathbf{5}}$ & $\mathbf{P}_{\mathbf{6}}$ & $\mathbf{P}_{\mathbf{7}}$ \\
\hline $\mathrm{P}_{1}$ & & 0.56 & 0.39 & 0.57 & 0.47 & 0.29 & 0.15 \\
\hline $\mathrm{P}_{2}$ & 0.15 & & 0.39 & 0.69 & 0.38 & 0.37 & 0.16 \\
\hline $\mathrm{P}_{3}$ & 0.27 & 0.30 & & 0.36 & 0.40 & 0.40 & 0.12 \\
\hline $\mathrm{P}_{4}$ & 0.21 & 0.23 & 0.36 & & 0.44 & 0.29 & 0.14 \\
\hline $\mathrm{P}_{5}$ & 0.23 & 0.36 & 0.56 & 0.42 & & 0.34 & 0.13 \\
\hline $\mathrm{P}_{6}$ & 0.33 & 0.15 & 0.17 & 0.31 & 0.23 & & 0.12 \\
\hline $\mathrm{P}_{7}$ & 0.22 & 0.01 & 0.13 & 0.09 & 0.22 & 0.10 & \\
\hline
\end{tabular}

The dendrogram generated from RAPD data separated the studied inbred lines into two main clusters as represented in Fig. 3A. The inbred line $\mathrm{P}_{7}$ was placed alone in the first main cluster. The second main cluster contained the rest of the inbred lines and this cluster separated into two sub-clusters; the first one grouped the inbred lines $\mathrm{P}_{3}$ and $\mathrm{P}_{6}$, while, the second sub-cluster divided into two sub-sub clusters; the first contained $\mathrm{P}_{5}$ and the second included $\mathrm{P}_{1}, \mathrm{P}_{2}$ and $\mathrm{P}_{4}$.

The dendrogram obtained with SSR markers (Fig. 3B), also divided the inbred lines into two main clusters. The inbred line $\mathrm{P}_{7}$ placed alone as in RAPD markers in the first main cluster. The second main cluster was divided into two sub-clusters; the first had the inbred lines $\mathrm{P}_{1}$ and $\mathrm{P}_{6}$, whereas, the second subcluster divided into two sub-sub clusters; the first contained $\mathrm{P}_{2}$ and the second included $\mathrm{P}_{3}, \mathrm{P}_{4}$ and $\mathrm{P}_{5}$. Both RAPD and SSR markers showed kind of similarity in the topology of their respective dendrograms. Even though, notable differences in the position of the inbred lines were observed, except the inbred line $\mathrm{P}_{7}$ which separated into out group in both methods (RAPD and SSR) that indicate this inbred is highly diverse than other maize inbreeds. 

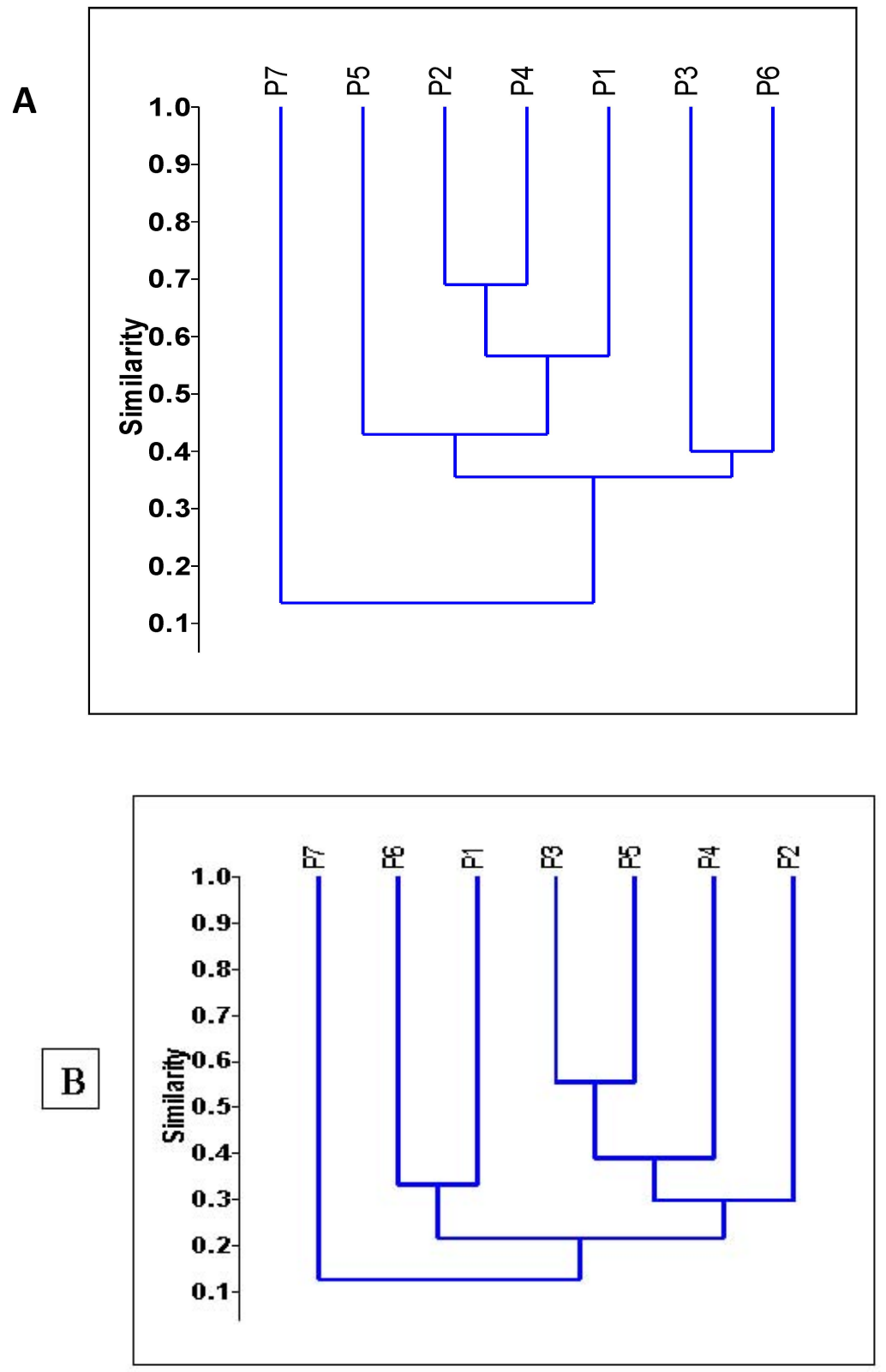

Fig. 3. Dendrogram generated based on UPGM clustering method using PAST program and Jacquard's coefficient using RAPD (A) and SSR (B) data among the seven inbred lines.

Egypt. J. Agron. 38, No. 3 (2016) 
Correlation between RAPD and SSR markers

The correlation coefficient $(r)$ between the similarity matrices obtained with RAPD and SSR markers was not significant $(\mathrm{r}=0.34, \mathrm{p}>0.05)$. These results are similar with those reported by Garcia et al. (2004), who observed a correlation of (0.33) between RAPD and SSR markers. Also, Cholastova et al. (2011) found that RAPD and SSR markers were poorly correlated (0.11) when studying genetic diversity in maize. However, Souza et al. (2008) and Leal et al. (2010) found high and significant correlations $(0.57$ and 0.55$)$ between RAPD and SSR markers in maize, respectively. The main reason for the limited correlation between RAPD and SSR markers might be the fact the RAPD markers are dominant, whereas, SSR are co-dominant. Moreover, the number of primers used and nature of genetic polymorphism detected by the two markers may affect the correlation between them (Pejic et al. 1998 and Sun et al., 2001).

Correlations of genetic distances with $F_{l}$ hybrids grain yield

Genetic distances (GD) based on each of RAPD and SSR markers were insignificantly correlated with $\mathrm{F}_{1}$ hybrids grain yield, the correlation values being $(\mathrm{r}=0.02$ and $0.429 \mathrm{P}>0.05)$, respectively. These results indicate that it is difficult to predict the $F_{1}$ hybrids grain yield from the genetic distance of the inbred lines used in this study. These results agree well with the findings of Menkir et al. (2010), Akaogu et al. (2012) and Oyekunle et al. (2015). They reported insignificant correlation between molecular marker-based GD and $\mathrm{F}_{1}$ hybrids grain yield. However, these results are contrary to the findings of Phumichai et al (2008) and Makumbi et al. (2011) who reported significant correlation between GD and $\mathrm{F}_{1}$ hybrids grain yield. The lack of correlation between GD and $F_{1}$ hybrids performance could be due to the absence of linkage between genes controlling the trait and markers used to estimate GD, inadequate genome coverage, random marker distribution and diversified effect of dominance (Bernardo, 1992).

\section{References}

Abd El-Mottalb, A.A., Mostafa, M.A. and Gameaa, H.Al.A. (2013) Combining ability estimates in some white maize inbred lines for yield and other traits. Egypt. J. Plant Breed. 17 (3), 13 - 22.

Abdellatif, K.F. and Khidr, Y.A. (2010) Genetic diversity of new maize hybrids based on SSR markers as compared with other molecular and biochemical markers. J. Crop Sci. Biotech. 13 (3), 139-145.

Abo El-Haress, S.M. (2015) Diallel analysis for yield, downy mildew and agronomic characters in maize (Zea mays L.). Alex. J. Agric. Res. 60 (1), 25-31.

Ahmed, M.F. (2013) Diallel analysis and biochemical genetic markers for heterosis and combining ability under two sowing dates of maize inbred lines. Asian J. Crop Sci. 5, 81-94.

Akaogu, I.C., Badu-Apraku, B., Adetimirin, V.O., Vroh-BI, I., Oyekunle, M. and Akinwale, R.O. (2012) Genetic diversity assessment of extra-early maturing yellow maize inbreds and hybrid performance in Striga-infested and Striga-free environments. J. Agric. Sci. 151, 519-537. 
Akinwale, R.O., Badu-Apraku, B., Fakorede, M.A.B. and Vroh-Bi, I. (2014) Heterotic grouping of tropical early-maturing maize inbred lines based on combining ability in Striga-infested and Striga-free environments and the use of SSR markers for genotyping. Field Crops Res. 156, 48-62.

Attia, A.N., Sultan, M.S., Badawi, M.A., Abdel-Moneam, M.A. and Al-Rawi, A.R.M. (2015) Estimation of combining ability and heterosis for some maize inbred lines and its single crosses. J. Plant Production, Mansoura Univ. 6 (1), 83 -98.

Badu-Apraku, B. and Oyekunle, M. (2012) Genetic analysis of grain yield and other traits of extra-early yellow maize inbreds and hybrid performance under contrasting environments. Field Crops Res. 129, 99-110.

Bernardo, R. (1992) Relationships between single-cross performance and molecular markers heterozygosity. Theor. Appl. Genet. 83, 6286-6234.

Bruel, D.C., Carpentieri-Pípolo, V., Ruas, C.F., Gerage, A.C. and Souza, S.G.H. (2007) Assessment of genetic diversity in maize inbred lines using RAPD markers. Crop Breeding and Applied Biotechnology, 7, 173-178.

Cholastova, T., Soldanova, M. and Pokorny, R. (2011) Random amplified polymorphic DNA (RAPD) and simple sequence repeat (SSR) marker efficiency for maize hybrid identification. African Journal of Biotechnology, 10 (24), 4794-4801.

Devi, P. and Singh, N.K. (2011) Heterosis, molecular diversity, combining ability and their interrelationships in short duration maize (Zea mays L.) across the environments. Euphytica, 178 (1), 71-81.

Dodiya, N.S. and Joshi, V.N. (2002) Gene action for grain yield and its attributes in maize (Zea mays L.) Indian J. Genet. and Plant Breed, 62, 253-254.

Doyle, J. and Doyle, J. (1990) Isolation of plant DNA from fresh tissue. Focus, 12 (1), 13-15.

El-Badawy, M.E.M. (2013) Heterosis and combining ability in maize using diallel crosses among seven new inbred lines. Asian J. Crop Sci. 5 (1), 1-13.

El-Gonemy, M.A.M. (2015) Combining ability of seven new white maize inbred lines for yield and some agronomic traits. Egypt. J. Plant Breed.19 (1), 15-24.

El-Shamarka, Sh.A., Abdel-Sattar, A. M. and El-Nahas, M.M. (2015) Heterosis and combining ability for yield and its components through diallel cross analysis in maize (Zea mays L.). Alex. J. Agric. Res. 60 (2), 87-94.

Garcia, A.A.F., Benchimol, L.L., Barbosa, A.M.M. and Geraldi, I.O. (2004) Comparison of RAPD, RFLP, AFLP and SSR markers for diversity studies in tropical maize inbred lines. Genet. Mol. Biol. 27, 579-588.

Griffing, B. (1956) Concept of general and specific combining ability in relation to diallel crossing systems. Aus. J. Biol. Sci. 9, 463-493. 
Hassan, M.A.A. (2015) Evaluation of new white maize hybrids for resistance to downy mildew and grain yield under different environments. J. Agric. Res. Kafrelsheikh Univ. 41, 565-575.

Hassan, M.A.A., El-Shenawy, A.A., Abo El-Hares, S.M. and Khalil, M.A.G. (2016) Combining ability of new yellow maize inbred lines for earliness and grain yield. Egypt. J. Plant Breed. 20 (2), $353-362$.

Hefny, M. (2010) Genetic control of flowering traits, yield and its components in maize (Zea mays L.) at different sowing dates. Asian J. Crop Sci. 2, 236-249.

Ibrahim, H.S. (2014) Estimation of genetic parameters for yield and its components and some growth characters in maize diallel crosses. Egypt. J. Plant Breed. 18 (3), 531 - 549.

Jaccard, P. (1908) Nouvelles recherches sur la distribution florale. Bul. Soc. Vaudoise Sci. Nat. 44, 223-270.

Kamara, M.M. (2016) Combining ability and genetic diversity using SSR markers for some maize inbred lines. Egypt. J. Plant Breed. 20 (2), 373-394.

Kamara, M.M. (2015) Diallel analysis of some yellow maize inbred lines under low and normal nitrogen levels. International J. Plant Breeding and Genetics, 9 (2), 32 - 43.

Kamara, M.M. and Rehan, M.R. (2015) Combining ability in maize under two nitrogen levels and assessing genetic diversity using RAPD marker. J. Plant Production, Mansoura Univ. 6 (12), 2051-2067.

Laborda, P.R., Oliveira, K.M., Garcia, A.A.F., Paterniani, M.E.A.G.Z. and Souza, A. P. (2005) Tropical maize germplasm: what can we say about its genetic diversity in the light of molecular markers? Theor. Appl. Genet.111, 1288-1299.

Lanza, L.L.B., Souza Jr, C.L., Ottoboni, L.M.N., Vieira, M.L.C. and Souza, A.P. (1997) Genetic distance of inbread lines and prediction of maize single-cross performance using RAPD markers. Theoretical and Applied Genetics, 94, 1023-1030.

Leal, A.A., Mangolin, C.A., do Amaral Júnior, A.T., Gonçalves, L.S.A., Scapim, C.A., Mott, A.S., Eloi, I.B.O., Cordovés, V. and da Silva, M.F.P. (2010) Effciency of RAPD versus SSR markers for determining genetic diversity among popcorn lines. Genetics and Molecular Research, 9 (1), 9-18.

Legesse, B.W., Myburg, A.A., Pixley, K.V. and Botha, A.M. (2007) Genetic diversity of African maize inbred lines revealed by SSR markers. Hereditas, 144, 10-17.

Makumbi, D., Betran, J.F., Ba“nziger, M. and Ribaut, J.M. (2011) Combining ability, heterosis and genetic diversity in tropical maize (Zea mays L.) under stress and nonstress conditions. Euphytica, 180,143-162.

Menkir, A., Melake-Berhan, A., Thé, C., Ingelbrecht, I. and Adepoju, A. (2004) Grouping of tropical mid-altitude maize inbred lines on the basis of yield data and molecular markers. Theor. Appl. Genet. 108, 1582-1590. 
Menkir, A., Adetimirin, V.O., Yallou, C.G. and Gedil, M. (2010) Relationship of genetic diversity of inbred lines with different reactions to Striga hermonthica (Del.) Benth and the performance of their crosses. Crop Sci. 50, 602-611.

Molin, D., Coelho, C.J., Máximo, D.S., Ferreira, F.S., Gardingo, J.R. and Matiello, R.R. (2013) Genetic diversity in the germplasm of tropical maize landraces determined using molecular markers. Genetics and Molecular Research, 12 (1), 99-114.

Mousa, S.Th.M. (2014) Diallel analysis for physiological traits and grain yield of seven white maize inbred lines. Alex. J. Agric. Res. 59, 9-17.

Mukharib, D.S., Patil, V.C., Biradar, D.P., Salimath, P.M. and Chimmad, V.P. (2010) Assessment of molecular diversity in selected maize inbreds. Karnataka Journal of Agricultural Sciences, 23, 409-412.

Nyaligwa, L., Hussein, S., Amelework, B. and Ghebrehiwot, H. (2015) Genetic diversity analysis of elite maize inbred lines of diverse sources using SSR markers. Maydica, 60, 1-8.

Oyekunle, M., Badu-Apraku, B., Hearne, S. and Franco, J. (2015) Genetic diversity of tropical early maturing maize inbreds and their performance in hybrid combinations under drought and optimum growing conditions. Field Crops Res. 170, 55-65.

Pandit, M., Chakraborty, M., Haider, Z.A., Pande, A., Sah, R.P. and Sourav, K. (2016) Genetic diversity assay of maize (Zea mays L.) inbreds based on morphometric traits and SSR markers. African Journal of Agricultural Research, 11(24), 2118-2128.

Pejic, I., Ajmone-Marsan, P., Morgante, M., Kozumplick, V., Castiglioni, P., Taramino, G. and Motto, M. (1998) Comparative analysis of genetic similarity among maize inbred lines detected by RFLPs, RAPDs, SSRs and AFLPs. Theor. Appl. Genet. 97, 1248-1255.

Phumichai, C., Doungchan, W., Puddhanon, P., Jampatong, S., Grudloyma, P., Kirdsri, C., Chunwongse, J. and Pulam, T. (2008) SSR-based and grain yield-based diversity of hybrid maize in Thailand. Field Crops Res. 108, 157-162.

Ranum, P., Pena-Rosas, J.P. and Garcia-Casal, M.N. (2014) Global maize production, utilization, and consumption. Annals of the New York Academy of Sciences, 1312, $105-112$.

Senior, M.L., Murphy, J.P., Goodman, M.M. and Stuber, C.W. (1998) Utility of SSRs for determining genetic similarities and relationships in maize using an agarose gel system. Crop Sci. 38, 1088-1098.

Souza, S.G.H., Pípolo, V.C., Ruas, C.F., Carvalho, V.P., Ruas, P.M. and Gerage, A.C. (2008) Comparative analysis of genetic diversity among the maize inbred lines (Zea mays L.) obtained by RAPD and SSR markers. Braz. Arch. Biol. Technol. 51 (1), 183-192.

Sserumaga, J.P., Makumbi, D., Ji, H., Njoroge, K., Muhomi, J.W., Chemining'wa, G.N., Si-myun, L., Asea, G. and Kim, H. (2014) Molecular characterization of tropical maize inbred lines using microsatellite DNA markers. Maydica, 59, 267-274.

Egypt. J. Agron. 38, No. 3 (2016) 
Steel, R.G.D. and Torrie, J.H. (1980) "Principles and Procedures of Statistics. A Biometrical Approach". $2^{\text {nd }}$ ed. Mc Graw Hill, N.Y., USA.

Sun, G.L., William, M., Liu, J., Kasha, K.J. and Pauls, K.P. (2001) Microsatellite and RAPD polymosphisms in Ontario corn hybrids are related to the commercial sources and maturity ratings. Molecular Breeding, 7, 13-24.

\title{
تحليل الهجن التبادلية والتباعد الوراثى لبعض سلالات من الذرة



\author{
مدحت رمضان ريحان و محمد محمد قمرة"

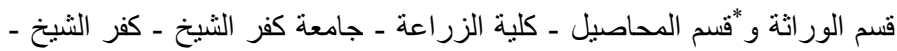

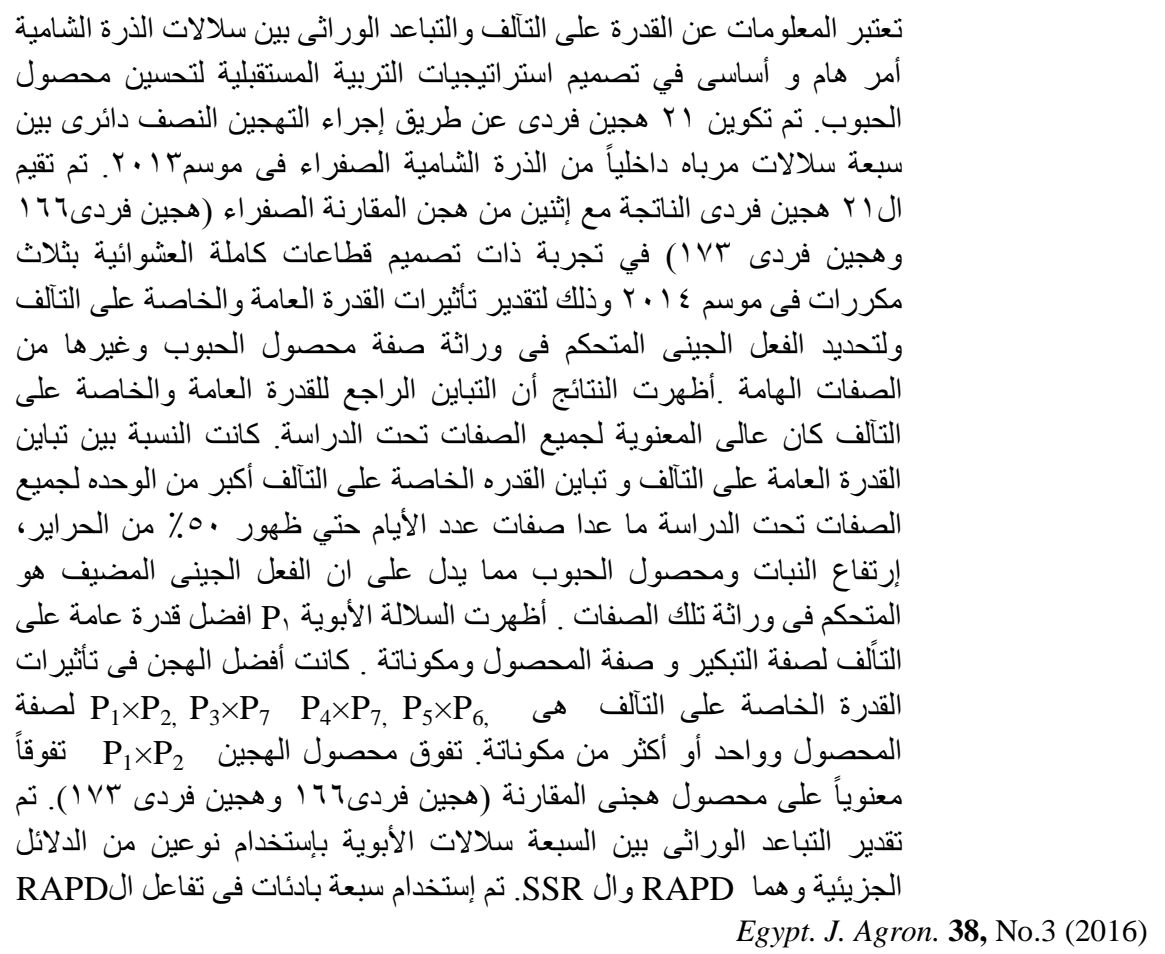




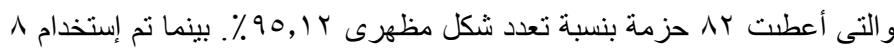



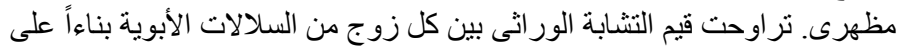

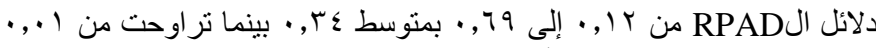

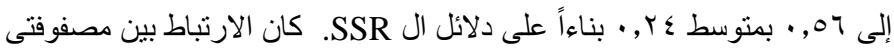

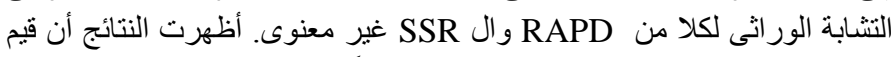

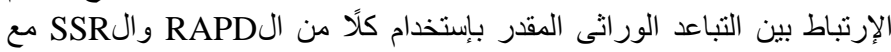

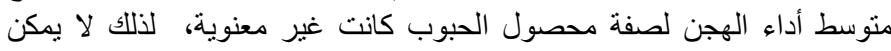



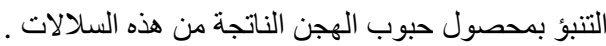

